\title{
GLP1 agonists beyond glycemic control_-redefining their role
}

\author{
Subhankar Chowdhury ${ }^{1}$. Soumik Goswami ${ }^{2}$
}

Published online: 14 October 2020

(C) Research Society for Study of Diabetes in India 2020

The "incretin effect" was discovered in 1964 when it was found that greater and more sustained insulin release occurs after oral glucose load compared to an equivalent intravenous load [1]. While the discovery of glucose-dependent insulinotropic peptide (GIP) provided the first etiological explanation for the "incretin effect," it was only later in the 1980s after the discovery of another incretin hormone - glucagon-like peptide 1 (GLP1) - that the entero-insular axis came into scientific limelight [2]. GLP1, released from the distal ileum and colon within minutes of a meal, was found to be more effective than GIP in effecting glucose-dependent insulin production and secretion. Subsequent studies showed that GLP1 also decreases glucagon secretion, increases glucose uptake and glycogen synthesis in peripheral tissues independent of insulin/ glucagon, delays gastric emptying, and increases satiety making scientists sit up and take notice of its therapeutic potential for diabetes management [3]. However, endogenous GLP1 is rapidly broken down by dipeptidyl peptidase-4 (DPP-4) and has a half-life of only 2 min, making it unsuitable as a pharmacotherapy for diabetes. The discovery of exendin-4 from the venom of the gila monster, Heloderma suspectum, turned things around as it acted like native GLP1 but was resistant to DPP-4 inhibition and possessed a much longer half-life [4]. Exenatide, a synthetic peptide identical to exendin-4, was the first GLP1 agonist to be approved by the US Food and Drug Administration (USFDA) in 2005 for the treatment of type 2 diabetes mellitus (T2DM) following which several others of this drug class were introduced given their unique profile of effective glucose lowering with low risk of hypoglycemia and the additional benefit of weight reduction.

Subhankar Chowdhury

subhankar.chowdhury@gmail.com

1 Department of Endocrinology and Metabolism, IPGME\&R and SSKM Hospital, 244 AJC Bose Road, Kolkata 700020, India

2 Department of Endocrinology, Nilratan Sircar Medical College, Kolkata, India
However, the beauty of the GLP1 agonist group of agents lies in their role beyond blood glucose lowering; this was subsequently discovered with the design and publication of several trials, most prominent of them being the USFDA-mandated cardiovascular outcome trials (CVOT) [5]. All the seven GLP1 agonist CVOTs completed thus far have shown non-inferiority to placebo, while liraglutide, subcutaneous semaglutide, albiglutide, and dulaglutide have also demonstrated significant reduction in composite cardiovascular (CV) outcomes. Three of the GLP1 agonist CVOTs also found favorable renal outcomes - in the LEADER trial, there were significantly fewer nephropathy events in the liraglutide group compared with placebo; in the SUSTAIN-6 trial, there were significantly fewer new or worsening nephropathy events using semaglutide compared with placebo; and the REWIND trial also showed significantly fewer adverse renal outcomes in the dulaglutide group compared with placebo [6]. Although the PIONEER 6 trial, the first CVOT for an oral GLP1 agonist (semaglutide), did not show cardiovascular benefit, this study was of a shorter duration with fewer participants compared to other trials so that fewer overall events were observed. A larger CVOT with oral semaglutide called "A Heart Disease Study of Semaglutide in Patients with Type 2 Diabetes" (SOUL) is currently in trial phase III [6]. The CVOTs were designed to maintain glycemic equipoise, and subgroup analysis revealed that the beneficial cardiovascular and renal effects noted with these agents occurred independent of blood glucose reduction. A systematic review and metaanalysis published in 2019 concluded that GLP1 agonists have beneficial effects on cardiovascular, kidney, and mortality outcomes in patients with T2DM. A more recent meta-analysis in 2020 showed significant reduction in major adverse CV events, $\mathrm{CV}$ and total mortality, stroke, and hospitalization for heart failure, with a trend for reduction of myocardial infarction in patients with T2DM with and without established CV disease [7, 8]. These findings have led to a paradigm shift in the management of T2DM with guidelines recommending that those with established cardiovascular disease should use either a GLP1 agonist (or SGLT2 inhibitor) with established cardiovascular benefit as the first add-on therapy to metformin irrespective of their glycated hemoglobin level [9]. 
Obesity pharmacotherapy is an area where GLP1 agonists have made their mark as well. GLP1 acts on the paraventricular and arcuate nuclei of the hypothalamus to suppress appetite [10]. They also act on other areas of the brain such as the mesolimbic system to diminish food-induced reward signals, thereby reducing food-seeking behavior [11]. GLP1 also acts on peripheral nervous system pathways to slow gastric emptying, and the resultant gastric stretch stimulates vagal afferent signals to the solitary nucleus of the medulla and onto the appetite centers of the hypothalamus to induce satiety or the area postrema to induce nausea [12]. An initial dose finding study with liraglutide showed the 3-mg dose to be most effective for inducing weight loss at 20 weeks and beyond [13]. This led to the Satiety and Clinical Adiposity-Liraglutide Evidence (SCALE) series of four trials which tested the effect of $3 \mathrm{mg}$ liraglutide on weight loss as an adjunct to diet and physical exercise. In composite data analysis of the SCALE trials, $3.0 \mathrm{mg}$ liraglutide led to a $7.5 \%$ weight loss over 1 year compared with $2.3 \%$ for placebo which was slightly better than naltrexone/ bupropion and lorcaserin and somewhat lesser than phentermine/topiramate [14]. In December 2014, $3 \mathrm{mg}$ liraglutide was approved by the USFDA as an adjunct to a reduced calorie diet and increased physical activity for chronic weight management in adults with obesity (BMI of $\geq 30 \mathrm{~kg} / \mathrm{m} 2$ ) or who are overweight (BMI of $\geq 27 \mathrm{~kg} / \mathrm{m} 2$ ) in the presence of at least one weight-related comorbid condition (e.g., hypertension, dyslipidemia, T2DM) [14].

GLP1 has also been attracting attention as a link between metabolic and neurodegenerative diseases on account of its influence on neurogenesis, neurodegeneration, retinal repair, and energy homeostasis [15]. GLP1 in animal models has been shown to improve learning and memory by modulating synaptic plasticity and reducing hippocampal neurodegeneration. Modulation of GLP1 activity has been demonstrated to influence amyloid b peptide aggregation in Alzheimer's disease (AD) and dopamine levels in Parkinson's disease (PD) in animal experiments. In animal PD models, GLP1 agonists were able to protect motor activity and dopaminergic neurons, whereas in $\mathrm{AD}$ models, they seemed to improve nearly all neuropathological features and cognitive functions. In a human trial with patients having moderate $\mathrm{PD}$, exenatide had a positive and sustained effect on clinically assessed motor function [16]. In animal models, GLP1 agonists have shown beneficial actions on brain ischemia, such as the reduction of cerebral infarct area and improvement of neurological deficit. Despite the substantial amount of animal data in this area, human trials are scanty although the possibilities are huge and the future promising.

In recent times, the domain where GLP1 agonists have generated considerable interest and evidence is that of nonalcoholic fatty liver disease (NAFLD). NAFLD is the most common chronic liver disease with a global prevalence of $25.2 \%$, and has a higher prevalence of $55.5 \%$ in T2DM [17]. NAFLD is divided into two histological subtypes of (a) nonalcoholic fatty liver (NAFL), characterized by isolated hepatic steatosis, often with mild nonspecific inflammation, and (b) nonalcoholic steatohepatitis (NASH), characterized by the presence of hepatic steatosis and hepatocellular injury with or without fibrosis [18]. NASH is the more severe form of NAFLD from where $20 \%$ can progress to cirrhosis, liver failure, and hepatocellular carcinoma. NASH in T2DM patients is associated with a higher risk of progressing to cirrhosis and hepatocellular carcinoma and the coexistence of NAFLD and T2DM is also related to increased risk of extrahepatic diseases like cardiovascular disease and chronic kidney disease [19]. Lifestyle modification leading to $5-10 \%$ decrease in body weight has shown significant improvement in hepatic steatosis, necroinflammation, and even fibrosis in NAFLD; but weight reduction rarely achieves complete resolution of NASH and is difficult to maintain over a long period of time [20]. NAFLD is closely associated with both hepatic and adipose tissue insulin resistance and GLP1 agonists have been shown to reduce insulin resistance [21]. GLP1 improves insulin signal transduction in adipocytes by upregulating Akt phosphorylation and protein expression of cyclins A, D1, and E and has been shown to activate genes involved in hepatic fatty acid oxidation and insulin sensitivity in hepatocytes isolated from rats with NASH [22]. The earliest clinical evidence of GLP1 agonist in NAFLD comes from studies where exenatide showed an improvement in hepatic enzymes [23]. Subsequently, there have been several studies with GLP1 agonists showing an improvement in alanine aminotransferase (ALT), aspartate aminotransferase (AST), and gamma-glutamyl transferase (GGT) in NAFLD making it the most commonly observed index in such studies [18]. Until date, seven studies with GLP1 agonists have shown significant reduction in liver fat content, the most recent of them being a study with dulaglutide in Indian patients with T2DM $[18,24]$. Although a handful of studies have shown significant improvement in noninvasive assessment of liver fibrosis in NAFLD with GLP1 agonists, their role for fibrosis regression and preventing progression to cirrhosis remains unclear [18]. Similarly, limited studies with GLP1 agonists have shown promise in improving histological features of NASH, but its role in management of NASH still remains inconclusive on account of scarcity of human studies with histological assessment [18].

The study by Zhang X et al. published in the current issue of the journal adds to the evidence on the role of GLP1 agonists in the management of NAFLD [25]. The authors looked at the effect of 3 months of liraglutide therapy on hepatic fat content in NAFLD in thirty-two patients with T2DM in China. The authors used fatty liver index (FLI) as a marker of hepatic fat on account of its ease of use and reliability which gives this study practical relevance [26]. None of the patients were on any hepatoprotective or lipidregulating drugs and the dose of antidiabetic drugs was unchanged during the duration of the trial to avoid confounding with the exception of liraglutide whose dose was uptitrated. There was a significant reduction in FLI by $26 \%$ and was accompanied by a significant decrease in AST and GGT as well by $7 \%$ and 39\%, respectively. Liraglutide also significantly improved body weight, serum triglyceride, insulin resistance, and blood glucose 
parameters in these patients but did not significantly improve ALT and other lipid parameters. While the finding of reduction in FLI which suggests a decrease in hepatic fat with liraglutide is interesting and provides further evidence of its potential to be a therapeutic option in NAFLD, these findings need to be replicated in appropriately powered larger randomized controlled trials looking at histological endpoints as well.

GLP1 agonists have come a long way since they were first made commercially available 15 years ago. Although initially launched as glucose lowering agents, their benefits beyond glycemic control have taken centerstage at present. They provide cardiorenal benefits and are effective agents to treat obesity. They have shown potential for the management of neurodegenerative disorders and more so in the management of NAFLD. If the evidence accumulated so far is a sign of things to come, the path ahead of GLP1 agonists for extra glycemic benefits looks bright and promising.

\section{References}

1. Elrick H, Stimmler L, Hlad CJ, et al. Plasma insulin response to oral and intravenous glucose administration. J Clin Endocrinol Metab. 1964;24:1076-82.

2. Kreymann B, Williams G, Ghatei MA, Bloom SR. Glucagon-like peptide-1 7-36: a physiological incretin in man. Lancet. 1987;2: 1300-4.

3. Drucker DJ, Nauck MA. The incretin system: glucagon-like peptide- 1 receptor agonists and dipeptidyl peptidase- 4 inhibitors in type 2 diabetes. Lancet. 2006;368:1696-705.

4. Eng J, Kleinman WA, Singh L, Singh G, Raufman JP. Isolation and characterization of exendin-4, an exendin-3 analogue, from Heloderma suspectum venom. Further evidence for an exendin receptor on dispersed acini from guinea pig pancreas. J Biol Chem. 1992;267(11):7402-5.

5. Food and Drug Administration. Diabetes mellitus-evaluating cardiovascular risk in new antidiabetic therapies to treat type 2 diabetes. Rockville: Food and Drug Administration; 2008.

6. Sheahan KH, Wahlberg EA, Gilbert MP. An overview of GLP-1 agonists and recent cardiovascular outcomes trials. Postgrad Med J. 2020;96(1133):156-61.

7. Kristensen SL, Rørth R, Jhund PS, Docherty KF, Sattar N, Preiss D, et al. Cardiovascular, mortality, and kidney outcomes with GLP-1 receptor agonists in patients with type 2 diabetes: a systematic review and meta-analysis of cardiovascular outcome trials [published correction appears in lancet diabetes Endocrinol. 2020 Mar;8(3): e2]. Lancet Diabetes Endocrinol. 2019;7(10):776-85.

8. Marsico F, Paolillo S, Gargiulo P, et al. Effects of glucagon-like peptide-1 receptor agonists on major cardiovascular events in patients with type 2 diabetes mellitus with or without established cardiovascular disease: a meta-analysis of randomized controlled trials [published online ahead of print, 2020 Feb 20]. Eur Heart J. 2020; ehaa082.

9. American Diabetes Association. 9. Pharmacologic approaches to glycemic treatment: standards of medical care in diabetes-2019. Diabetes Care. 2019;42:S90-102.

10. Shughrue P, Lane M, Mercenthaler I. Glucagon-like peptide-1 receptor (GLP1-R) mRNA in the rat hypothalamus. Endocrinology. 1996;137:5159-62.
11. Dickson S, Shirazi R, Hansson C, et al. The glucagon like peptide 1 (GLP-1) analogue, exendin-4, decreases the rewarding value of food: a new role for mesolimbicGLP-1 receptors. J Neurosci. 2012;32:4812-20.

12. Delgado-Aro S, Kim D, Burton D, et al. Effect of GLP-1 on gastric volume, emptying, maximum volume ingested, and postprandial symptoms in humans. Am J Physiol Gastrointest Liver Physiol. 2002;282:G424-31.

13. Astrup A, Rossner S, Van Gaal L, et al. Effects of liraglutide in the treatment of obesity: a randomised, double-blind, placebo controlled study. Lancet. 2009;374:1606-16.

14. Crane J, McGowan B. The GLP-1 agonist, liraglutide, as a pharmacotherapy for obesity. Ther Adv Chron Dis. 2016;7(2):92-107.

15. Grieco M, Giorgi A, Gentile MC, d'Erme M, Morano S, Maras B, et al. Glucagon-like peptide-1: a focus on neurodegenerative diseases. Front Neurosci. 2019;13:1112.

16. Athauda D, Maclagan K, Skene SS, Bajwa-Joseph M, Letchford D, Chowdhury K, et al. Exenatide once weekly versus placebo in Parkinson's disease: a randomised, double-blind, placebocontrolled trial. Lancet. 2017;390:1664-75.

17. Younossi ZM, Golabi P, de Avila L, Paik JM, Srishord M, Fukui N, et al. The global epidemiology of NAFLD and NASH in patients with type 2 diabetes: a systematic review and meta-analysis. J Hepatol. 2019;71(4):793-801.

18. Lv X, Dong Y, Hu L, et al. Glucagon-like peptide-1 receptor agonists (GLP-1 RAs) for the management of nonalcoholic fatty liver disease (NAFLD): a systematic review. Endocrinol Diab Metab. 2020;3:e0163.

19. Arrese M, Barrera F, Triantafilo N, Arab JP. Concurrent nonalcoholic fatty liver disease and type 2 diabetes: diagnostic and therapeutic considerations. Expert Rev Gastroenterol Hepatol. 2019;13(9):849-66.

20. Hannah WN, Harrison SA. Lifestyle and dietary interventions in the management of nonalcoholic fatty liver disease. Dig Dis Sci. 2016;61(5):1365-74.

21. Khan RS, Bril F, Cusi K, Newsome PN. Modulation of insulin resistance in nonalcoholic fatty liver disease. Hepatology (Baltimore, MD). 2019;70(2):711-24.

22. Svegliati-Baroni G, Saccomanno S, Rychlicki C, Agostinelli L, de Minicis S, Candelaresi C, et al. Glucagon-likepeptide-1 receptor activation stimulates hepatic lipid oxidation and restores hepatic signalling alteration induced by a high-fat diet in nonalcoholic steatohepatitis. Liver Int. 2011;31(9):1285-97.

23. Buse JB, Klonoff DC, Nielsen LL, Guan X, Bowlus CL, Holcombe $\mathrm{JH}$, et al. Metabolic effects of two years of exenatide treatment on diabetes, obesity, and hepatic biomarkers in patients with type 2 diabetes: an interim analysis of data from the open-label, uncontrolled extension of three double-blind, placebo-controlled trials. Clin Ther. 2007;29(1):139-53.

24. Kuchay MS, Krishan S, Mishra SK, et al. Effect of dulaglutide on liver fat in patients with type 2 diabetes and NAFLD: randomised controlled trial (D-LIFT trial) [published online ahead of print, 2020 Aug 31]. Diabetologia. 2020. https://doi.org/10.1007/s00125-020-05265-7.

25. Zhang X, Ba R, Jia Y, et al. The effect of liraglutide on nonalcoholic fatty liver disease in type 2 diabetes mellitus. Int J Diabetes Dev Ctries. 2020. https://doi.org/10.1007/s13410-020-00857-w.

26. Cuthbertson DJ, Weickert MO, Lythgoe D, Sprung VS, Dobson R, Shoajee-Moradie F, et al. External validation of the fatty liver index and lipid accumulation product indices, using $1 \mathrm{H}$-magnetic resonance spectroscopy, to identify hepatic steatosis in healthy controls and obese, insulin-resistant individuals. Eur J Endocrinol. 2014;171(5):561-9.

Publisher's note Springer Nature remains neutral with regard to jurisdictional claims in published maps and institutional affiliations. 